\title{
Reasoning about curvilinear motion: Using principles or analogy
}

\author{
RICHARD CATRAMBONE \\ Georgia Institute of Technology, Atlanta, Georgia \\ CAREN M. JONES \\ University of Massachusetts, Amherst, Massachusetts \\ and \\ JOHN JONIDES and COLLEEN SEIFERT \\ University of Michigan, Ann Arbor, Michigan
}

\begin{abstract}
People possess implicit theories about the motion of objects, theories that are often incorrect. When asked to predict the path of an object emerging from a curved tube, for example, people often say that the object will continue to follow a curved path. However, when solving a problem that reminds them of a familiar previous instance, people often reason by analogy to the instance. In this study, we show that a previous instance must be very superficially similar to a problem in order to be used as an analogy; otherwise, people will use their implicit theories as the basis of their reasoning.
\end{abstract}

The laws that govern the motion of objects seem to be enigmatic to many people. McCloskey and his colleagues have argued that people frequently account for their judgments about motion in nonfamiliar situations with theories akin to the impetus theory of the medieval period (McCloskey, Caramazza, \& Green, 1980; McCloskey \& Kohl, 1983; McCloskey, Washburn, \& Felch, 1983). According to the impetus theory, when an object is set in motion, it gains a force in the direction of its movement, and this impetus gradually dissipates. An object set in motion on a curved path, then, gains a curvilinear impetus that dissipates over time. Thus, when asked to predict the path of a ball emerging from a curved tube, people often say that the ball will continue to follow a curved path, in violation of Newton's law that a moving body with no force applied to it will move in a straight line (e.g., Kaiser, Jonides, \& Alexander, 1986; McCloskey et al., 1980). Such evidence has led to the conclusion that people's reasoning about motion is frequently governed by intuitive theories of motion. By this account, people are often incorrect in their judgments because their theories are naive and incorrect.

How, then, can people correctly accomplish everyday tasks such as washing a car with a length of curved garden hose? Just like the curved-tube problem, this task re-

This research was supported by Office of Naval Research Grant N00014-91-J-1137. Experiment 1 was presented at the 33rd Annual Meeting of the Psychonomic Society in November 1992. We thank Peter Dixon, Michael McCloskey, and an anonymous reviewer for helpful comments on an earlier draft of this paper, and Julie Thomas for help in collecting and coding the data in Experiment 2. Correspondence should be addressed to R. Catrambone, School of Psychology, Georgia Institute of Technology, Atlanta, GA 30332. quires a prediction about the path of a moving object that is no longer constrained by an obvious regulator, the hose. Yet in this case, informal observation tells us that the person washing the car will invariably aim the nozzle of the hose at the spot to be washed, rather than aiming off-target and expecting the water to curve. ${ }^{1}$ Consistent with this observation is the finding of Kaiser et al. (1986) that subjects predict a straight path for water in the curved-tube problem (even though these same subjects predict a curved path for a ball leaving the tube).

We suggest that in the Kaiser et al. (1986) study, those subjects who solved the water problem but failed to solve the ball problem were using different strategies on the two problems. When solving the water problem, they may have been reminded of using a hose to wash a car or water a lawn, and may have used that analog to predict the path of the water; when solving the ball problem, however, they were not reminded of any particular instance and, thus, may have relied on their (faulty) knowledge of physics.

\section{Reminding}

It may at first seem curious that subjects might use the garden-hose analogy for the water problem but not for the ball problem. Prior work has made it clear that learners possess a strong desire to use examples when solving new problems (Pirolli \& Anderson, 1985). Some studies for instance, have shown that learners frequently mention examples when solving a problem (e.g., Chi, Bassok, Lewis, Reimann, \& Glaser, 1989; Lancaster \& Kolodner, 1988; Ross, 1984) and that they will even follow an example rather than instructions if the two conflict (LeFevre \& Dixon, 1986). The preference for using examples thus seems quite strong. 
The analogical-reasoning literature has demonstrated that superficial features of analogs play a large role in determining whether an analog will be retrieved when solving a target problem (Catrambone \& Holyoak, 1989; Gick \& Holyoak, 1983; Holyoak \& Koh, 1987; Ross, 1984); that is, an example is more likely to be retrieved if it has surface objects that are similar to those in the target problem.

A number of theories, instantiated as computer models, exist in the analogical-reasoning literature to explain why a person is reminded of, or accesses, particular prior cases when working on a problem (e.g., ARCS [Thagard, Holyoak, Nelson, \& Gochfeld, 1990], MAC/FAC [Gentner, Rattermann, \& Forbus, 1993], and PUPS [Anderson $\&$ Thompson, 1989]). These models offer ways of calculating similarity as a function of the matches that occur between a representation of the problem and the example in memory. The details of this process vary among the models and are not relevant to the current paper; the important point, however, is that they agree that superficial features play a strong role in accessing analogs.

The problems used in this paper are essentially onestep problems, like those used by Kaiser et al. (1986). Thus, it seems likely that if a subject accesses an appropriate example - one, that is, with a solution that is correct for the current situation-the subject is likely to give the correct answer to the problem.

\section{EXPERIMENT 1}

Kaiser et al. (1986) found that subjects predicted a straight path for water emerging from a curved tube, but a curved path for a ball coming out of the same curved tube. These two situations differed not only in terms of what was traveling through the tube (i.e., water vs. ball), but also in the potentially important fact that in the water problem, the subjects were asked to imagine a hose being attached to the tube and then having the water go through the tube, whereas in the ball problem, there was no mention of a hose. Perhaps the mention of a hose was critical for subjects to access the garden-hose analog.

Experiment 1 tests the hypothesis that performance on the water problem is affected by whether or not a hose is mentioned. If a hose is mentioned, the subjects should be more likely to access the garden-hose analog and, therefore, to predict a straight path for the water when it emerges from the curved tube. If a hose is not mentioned, the subjects should be less likely to access the garden-hose analog and, therefore, more likely to use their intuitive theories about motion to generate their answers. On the basis of prior research (e.g., McCloskey, et al., 1980), it was expected that the latter subjects, who reasoned by their (erroneous) theories, would be more likely to predict a curved path for the water.

\footnotetext{
Method

Subjects. The subjects were 40 University of Michigan undergraduates ( 20 males, 20 females), who volunteered to be in the ex-
}

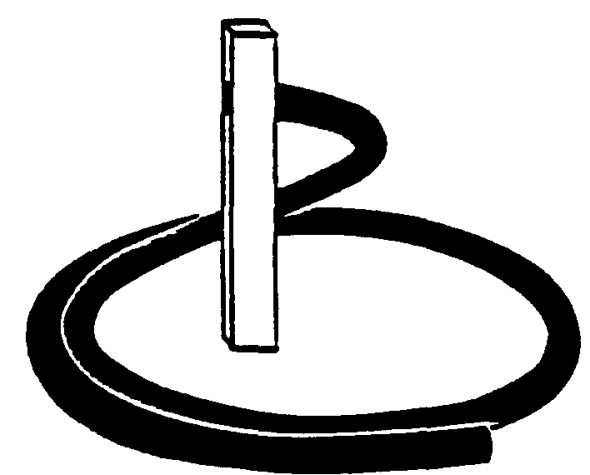

Figure 1. Spiral-tube apparatus.

periment (in exchange for a token payment) as they passed by the experiment station located in a busy hallway in a university building.

Materials and Procedure. The subjects were shown a clear plastic spiral tube mounted on a $2 \times 2.5-\mathrm{ft}$ plywood board that rested on a level table (see Figure 1). The $3 / 4$ in.-diam tube formed a spiral of $540^{\circ}$ rotation with an interior diam of 10 in. One end of the tube was raised $5 \mathrm{in}$. above the surface and was held in place by a piece of wood. There was a cup of water by the board.

The subjects were run individually and were randomly assigned to one of two conditions - "hose" and "no hose"-with the constraint that there were equal numbers of males and females in each condition. Subjects in the hose condition were read the following statement by the experimenter: "Suppose I attach a hose to the tube up here [experimenter points to elevated end of tube], so that the water comes out down here [experimenter points to lower end of tube]." Subjects in the no-hose condition were read the following statement by the experimenter: "Suppose I send this water [experimenter points to cup of water] through the tube up here [experimenter points to elevated end of tube], so that the water comes out down here [experimenter points to lower end of tube]."

After the subjects had heard one or the other of the above statements, the experimenter placed a piece of paper under the nonelevated end of the tube and the subjects were asked to draw on the paper the path that they thought the water would take when it exited the tube. After they had drawn the path, the subjects were given a piece of paper with a set of nine diagrams (see Figure 2), each of which was a schematic overhead view of the tube apparatus with a line or curve representing a path that an emerging object might take. The subjects were asked to select the diagram that showed the path that most closely matched the path he or she had just drawn. Their responses were coded by their diagram selections rather than by their drawings. The frequency with which the groups produced correct path predictions was analyzed using the likelihood-ratio chi-square test $\left(G^{2}\right.$; Bishop, Fienberg, \& Holland, 1975).

\section{Results and Discussion}

Of the subjects in the hose condition, $75 \%$ (15 of 20) indicated that the water would follow the correct straight path for the hose problem; by contrast, only $30 \%$ of the subjects in the no-hose condition (6 of 20) gave the correct answer. This is a statistically reliable difference $\left[G^{2}(1)=8.42, p=.004\right]$.

Mention of the word "hose" improved performance remarkably, presumably by making the subjects more likely to access the garden-hose analog and to base their path prediction on it. While the results of this experiment are compelling, it should be noted that the physics 

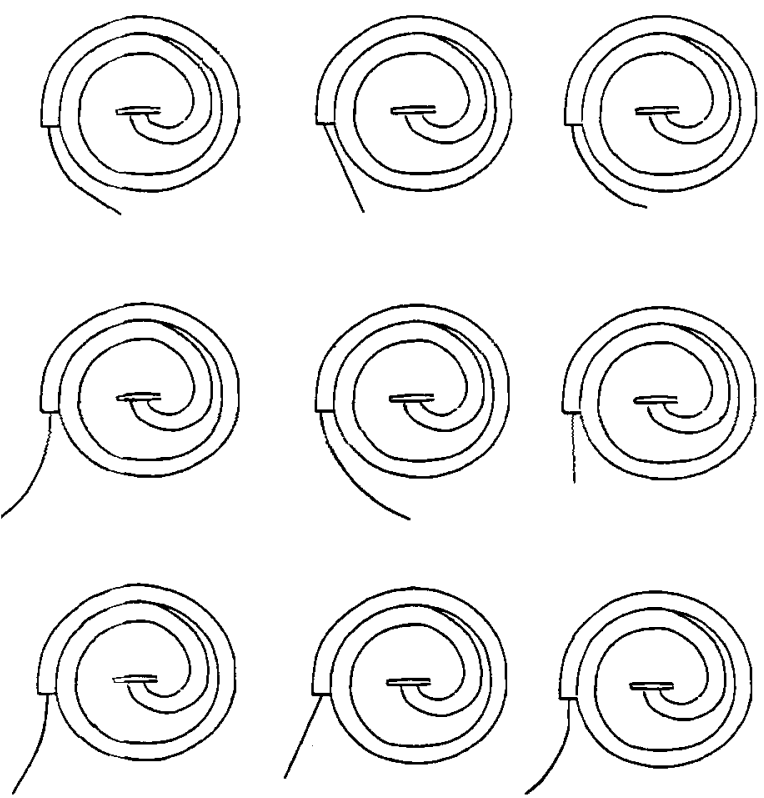

Figure 2. Set of path options for water emerging from spiral tube.

backgrounds of the subjects were not recorded. Although the subjects were randomly assigned to conditions, it is possible that an unequal distribution of physics knowledge between the two groups could have affected the results. In addition, the large effect for such a minimal manipulation (i.e., mentioning "hose" in the problem description) made it seem prudent to attempt a replication.

\section{EXPERIMENT 2}

Experiment 2 was a replication of Experiment 1, although this time, the physics background of each subject was recorded.

\section{Method}

Subjects. The subjects were 83 Georgia Institute of Technology undergraduates ( 54 males, 29 females), who participated in the experiment in exchange for extra credit in their psychology class. They all met the condition that they had taken no more than one physics course in high school and no more than one physics class in college.

Materials and Procedure. The materials and procedure were essentially identical to those used in Experiment l, with two differences: (1) the subjects provided evidence of their physics background (as a check that they followed the requirements for volunteering to be in the experiment); and (2) the experiment was conducted in a quiet room rather than in a busy hallway.

The subjects were randomly assigned to the hose $(n=41)$ and no-hose $(n=42)$ conditions. The groups were composed similarly with respect to physics background: $93 \%$ of the hose-condition subjects had one high school physics course and $85 \%$ had one college physics class, while for subjects in the no-hose condition, the percentages were $95 \%$ and $74 \%$, respectively.

\section{Results and Discussion}

The results were similar to those obtained in Experiment $1: 71 \%$ of the hose-condition-subjects produced the correct path, while only $38 \%$ of the subjects in the no-hose condition did so. This is a reliable difference $\left[G^{2}(1)=\right.$ $9.08, p=.003$ ]. It is a testament to the strength of the subjects' naive theories that so few subjects in the nohose condition produced the correct path, despite so many of them having one college-level physics class.

In each experiment, several subjects commented that they believed the speed of the water played a role in the direction it would take when exiting the spiral tube. They believed that fast-moving water would exit with a straighter path than water moving more slowly because the fast-moving water would have less of a tendency to "pick up" curvilinear momentum. If most of the subjects in Experiments 1 and 2 shared this belief, it is possible to argue that the hose-condition subjects might, like those in the no-hose condition, have been reasoning from an impetus theory of motion, but the greater implied speed of the water in the hose problem (compared with that in the no-hose version) might have led this theory to generate the correct answer more frequently,

In order to examine this possibility, another experiment was conducted, in which hoses were not mentioned, thus making the subjects more likely to reason by their theories of motion. Speed, however, was explicitly mentioned; if subjects reason by an impetus theory in this situation, and if speed is part of the theory, path predictions should vary as a function of speed. More specifically, those subjects dealing with a higher speed should be more likely to predict a straight path than those dealing with a lower speed.

\section{EXPERIMENT 3}

In this experiment, the imagined speed of the water moving through the tube was explicitly manipulated. Half of the subjects were told that the water was moving rapidly, and half were told that it was moving slowly. If speed is typically a factor in an intuitive theory of motion, and if the speed of an object influences its path as predicted by such a theory, these two groups of subjects should each predict different paths for the water.

On the premise that perhaps features of the material moving through the tube also affect path predictions, an additional factor was also examined in Experiment 3. It was conjectured that different qualities of objects (liquid vs. solid, easily moving vs. less-easily moving) might also be factors in people's theories of motion. To investigate this issue, some subjects were asked to make path predictions about substances other than water.

It is not entirely clear what effect the substance manipulation should have. One possibility is that the more similar the substance is to water, the more likely it is that the subjects' path predictions will be similar to the water-path prediction; thus, coffee and molasses should be given paths similar to that of water. Another possibility is that the more easily the substance moves through the tube (e.g., a steel ball and coffee move more easily than a hexagonal nut or molasses), the more likely it is that the response will be similar to the water response. 
Either of these outcomes would shed some light on the factors that affect people's determination of similarity for motion tasks.

If performance across the various conditions does not vary, and if the performance in these conditions is similar to that of the no-hose groups in Experiments 1 and 2, this would provide some support for the claim that the subjects in the hose conditions in Experiments 1 and 2 were frequently reasoning by analogy rather than by an impetus theory that took speed into account.

\footnotetext{
Method

Subjects. The subjects were 200 University of Michigan undergraduates ( 100 males, 100 females), who were recruited in the same manner as those in Experiment 1.

Materials and Procedure. The materials and procedure were similar to those used in the no-hose condition of Experiment 2. The subjects were asked to predict the paths of one of several materials emerging from the hose. The materials used were water (liquid, moves easily), coffee (liquid, moves easily), molasses (liquid, does not move easily), a steel ball (solid, moves easily), and a hexagonal nut (solid, does not move easily).

The speed of the material moving through the tube was stated explicitly. The following wording was used in the instructions read by the experimenter: "Suppose I send this [material] through this tube at a very [Value 1] speed so that it comes out very [Value 2] down here." The options for material in the instructions were water, coffee, molasses, a steel ball, or a hexagonal nut. The options for Value 1 were "low" or "high," while those for Value 2 were "slowly" or "rapidly."

The subjects in the liquid conditions had a cup of the appropriate liquid (water, coffee, or molasses) in view when making their path prediction. Likewise, the subjects in the solid conditions had an example of the appropriate object (a steel ball or a hexagonal nut) in view. The steel ball and hexagonal nut were each small enough to fit easily into the tube, though no substance or object was ever actually placed in the tube.

The 200 subjects were randomly and evenly assigned to one of 10 conditions defined by the speed and type of material moving through the tube.
}

\section{Results and Discussion}

Table 1 shows, for each condition, the proportion of subjects that predicted the correct path. Subjects were no more likely to predict a straight path when the speed was fast than they were when it was slow [38\% vs. $40 \%$; $\left.G^{2}(1)=.084, p=.77\right]$. Neither did type of substance reliably affect subjects' predictions, as accuracy ranged only between $35 \%$ (for molasses) and $42 \%$ [for water and the nut; $\left.G^{2}(4)=.757, p=.94\right]$. Although there is a danger in reasoning from null results, this study had sufficient power to detect a medium-sized effect at least $80 \%$ of the time with $\alpha$ set at .05 (as described in Cohen, 1992). For instance, if the fast-versus-slow difference had been $13 \%$ or greater (assuming that one of the groups was at the $38 \%-40 \%$-correct level), the $G^{2}$ analysis would have detected a difference at the .05 level. It seems reasonable to claim, then, that there was a good chance of detecting an effect if there had been one. Thus, we are reasonably confident that neither speed nor substance influenced subjects' predictions.

Overall, the subjects were correct $39 \%$ of the time. This is similar to the performance of the subjects in the no-hose condition in the prior experiments (30\% in Experiment 1 and $38 \%$ in Experiment 2).

The results are consistent with at least two explanations. First, it is possible that the subjects in the no-hose condition in each experiment were usually reasoning from their intuitive theories of motion and therefore tended to predict the wrong path, while the subjects in the hose condition were usually reasoning from an analogy, such as the garden-hose analogy, and therefore tended to predict the correct path.

Alternatively, the subjects in both conditions may have been reasoning from naive theories of motion, but speed and substance are typically not factors in their theories; instead, some other feature of the "hose" situation might have affected how the subjects used their naive theories in making path predictions. In this explanation, the fact that the speed and substance manipulations had no effect on performance does not imply that the subjects in the hose condition were reasoning by analogy.

Indirect support for the claim that subjects in the nohose condition were reasoning by intuitive theories comes from the work of McCloskey et al. (1980), who found that increasing the curvature of the tube in which a ball was traveling led subjects to predict increasingly curved paths for the ball when it emerged from the tube. On the basis of interview data with subjects, McCloskey et al. suggested that the subjects predicted increasingly curved paths because they believed that the ball acquired more curvilinear impetus as a function of the curvature of the tube.

\section{EXPERIMENT 4}

The results from prior studies and from the first three experiments reported here do not allow a discrimination to be made between the two explanations offered above for why the subjects in the hose condition outperform those in the no-hose condition. In order to examine more directly whether the subjects in the hose condition were reasoning from the garden-hose analogy more often those those in the no-hose condition, the subjects in Experiment 4 were asked how they produced their path predictions and, secondarily, whether they were reminded of anything when they were making their predictions. If the subjects in the hose condition were reasoning by analogy while those in the no-hose condition were reasoning with principles of motion, this difference might be reflected in their responses. Furthermore, subjects'

Table 1

Percentage of Subjects Predicting Correct Path for Each of the 10 Conditions of Experiment 3

\begin{tabular}{lcccccc}
\hline & \multicolumn{5}{c}{ Substance } & \\
\cline { 2 - 6 } \multicolumn{1}{c}{ Speed } & Water & Coffee & Molasses & Ball & Nut & Average \\
\hline Fast & 45 & 35 & 35 & 40 & 35 & 38 \\
Slow & 40 & 40 & 35 & 35 & 50 & 40 \\
Average & 42 & 38 & 35 & 38 & 42 & 39 \\
\hline
\end{tabular}

Note-There were 20 subjects in each condition. 
responses to the questions might predict the correctness of their path predictions.

\section{Method}

Subjects. The subjects were 50 Georgia Institute of Technology undergraduates ( 32 males, 18 females), who participated in exchange for extra credit in their psychology class. None of them had taken more than one physics course in high school and one physics class in college, a condition of participating in the experiment.

Materials and Procedure. The materials and procedure were essentially identical to those used in Experiment 2, except that after producing their path predictions, the subjects were asked the following series of questions:

Question 1. How did you solve this problem?

Question 2. While you were working on the problem, did it remind you of anything?

Question 3. Were you reminded of any particular kind of hose when you were trying to solve the problem?

Question 1 was designed to be nonleading, while Questions 2 and 3 were increasingly more direct and leading. Questioning stopped if the subject produced the garden-hose analog.

The subjects' responses to the questions were scored in the following manner: For Question 1, responses were categorized as (1) mentioning garden hose, (2) mentioning theory or guessing, or (3) mentioning some other analog; for Question 2, they were categorized as (1) mentioning garden hose, (2) mentioning some other analog, or (3) not reminded of anything; and for Question 3, responses were categorized as (1) garden hose or (2) not garden hose.

Subjects were randomly assigned to the hose $(n=25)$ and no-hose $(n=25)$ conditions. The composition of the groups with respect to physics background was similar: $84 \%$ of the hose-condition subjects had one high school physics course and $40 \%$ had one college physics class; for the subjects in the no-hose condition, the percentages were $80 \%$ and $48 \%$, respectively.

\section{Results and Discussion}

The subjects in the hose condition predicted the correct path more often than those in the no-hose condition did $\left[76 \%\right.$ vs. $\left.48 \% ; G^{2}(1)=4.24, p=.04\right]$.

As can be seen from Table 2, in response to Question 1, over half of the subjects in the hose condition mentioned the garden-hose analogy, while none of the subjects in the no-hose condition did. This difference is significant $\left[G^{2}(2)=21.79, p<.0001\right]$. Table 2 also shows that, once again, the hose-condition subjects responded to the leading questions (Questions 2 and 3) with the garden-hose analogy more often than the subjects in the no-hose condition did [Question 2: $G^{2}(2)=$ 19.76, $p<.0001$; Question 3: $G^{2}(1)=36.95, p<.0001$ ]

Finally, the subjects' path predictions were compared as a function of their responses to Question 1. All of the subjects who mentioned the garden-hose analogy (i.e., the 15 hose-condition subjects) or some other concrete example (i.e., 2 hose-condition and 3 no-hose condition subjects) produced the correct response, while $37 \%$ of the subjects who said that they either used a theory or guessed (i.e., 11 of 30 ) produced the correct response. This difference is significant $\left[G^{2}(2)=26.98, p<.0001\right]$, which demonstrates that the 15 subjects in the hose condition the who used garden-hose analogy all predicted the correct path. Interestingly, the 5 subjects who men- tioned other analogs also predicted the correct path; these included 1 subject who mentioned swinging a tether, an appropriate analogy.

In response to Question 2, an increased number of subjects in the no-hose condition mentioned an analog other than the garden-hose analog. However, responses to this question, as well as those to Question 3, must be interpreted more cautiously than responses to Question 1, since the subjects may have been led to provide responses that did not accurately reflect their reasoning concerning the path-prediction problem.

\section{GENERAL DISCUSSION}

The results from Experiments 1 and 2 are consistent with the claim that people will use an analogy or prior instance in their reasoning when attempting to solve a new problem, but that strong cues are needed to remind the problem solver of the prior problem. Experiment 4 provided converging evidence for this claim by demonstrating that the subjects in the hose condition were far more likely than those in the no-hose condition to report thinking of the garden-hose analogy when solving the path-prediction problem. In many classroom and realworld situations, potentially relevant prior examples are often easily accessed due to the learning context. For example, if one is working on an algebraic word problem at the end of a chapter, one will be likely to be reminded of prior examples from the chapter because these examples are connected to the current context; when these powerful contextual cues are absent, however, it appears that learners are much less likely to access relevant examples or analogs.

It is worth noting that the problem-solving literature has documented many cases in which learners will access previously studied, but inappropriate, examples when working on new problems. That is, they access ex-

Table 2

Percentage of Subjects Producing Different Responses to Questions 1-3 (Experiment 4)

\begin{tabular}{lcc}
\hline & \multicolumn{2}{c}{ Group } \\
\cline { 2 - 3 } \multicolumn{1}{c}{ Answer } & Hose $(n=25)$ & No Hose $(n=25)$ \\
\hline Garden hose & 60 & 0 \\
Theory/guessing & 32 & 88 \\
Other analogy & 8 & 12 \\
& Question 2 & \\
Garden hose & 60 & 4 \\
Other analogy & 8 & 52 \\
No reminding & 32 & 84 \\
& 92 & 12 \\
Garden hose & 8 & 88 \\
Not garden hose & Question 3 & \\
\hline
\end{tabular}

Note-The percentages reported for Questions 2 and 3 are cumulative. For instance, the $92 \%$ of the hose-condition subjects who reported thinking of a garden hose in response to Question 3 also includes those subjects who mentioned thinking of a garden hose in response to Question 1 or Question 2. 
amples that are superficially similar to the problem but that do not share the same solution or solution structure (Reed, Ackinclose, \& Voss, 1990; Ross, 1987, 1989). For example, Ranney and Thagard (1988; see also Caramazza, McCloskey, \& Green, 1981) reported that some people predicted that a pendulum bob would follow a curved path, rather than falling straight down, if it was released at the top of the pendulum's arc. This answer was partially based on people's erroneous recall of children jumping from swings; they seemed to forget, or not notice, that children jump before the swing reaches the top of its arc.

While the present study focused primarily on a single problem - that of predicting the path of water through a tube - the results are consistent with the analogicalreasoning and problem-solving literatures demonstrating the powerful effect of surface cues on the likelihood of people accessing a particular exemplar (e.g., Gentner et al., 1993; Reed et al., 1990; Ross, 1984, 1987, 1989). The present results extend this finding into the domain of reasoning about motion.

Thus, our results speak to two literatures. With respect to the analogical-reasoning literature, they are consistent with the observation that surface cues have a strong effect on memory access and thus on subsequent problem solving. In our experiments, the important cue seemed to be an explicit mention of a hose (or perhaps the interaction of water and mention of a hose). With respect to the "naive physics" literature, our results suggest that people will often rely on their intuitive theories to predict motion, but that if they are reminded of a familiar analog, they will reason from it rather than from their naive theories.

\section{REFERENCES}

ANDERSON, J. R., \& THOMPSON, R. (1989). Use of analogy in a production system architecture. In S. Vosniadou \& A. Ortony (Eds.), Similarity and analogical reasoning (pp. 267-297). Cambridge: Cambridge University Press.

Bishop, Y. M. M., FienberG, S. E., \& Holland, P. W. (1975). Discrete multivariate analysis: Theory and practice. Cambridge, MA: MIT Press.

Caramazza, A., McCloskey, M., \& Green, B. (1981). Naive beliefs in "sophjsticated" subjects: Misconceptions about trajectories of objects. Cognition, 9, 117-123.

Catrambone, R., \& Holyoak, K. J. (1989). Overcoming contextual limitations on problem-solving transfer. Journal of Experimental Psychology: Learning, Memory, \& Cognition, 15, 1147-1156.

Chi, M. T. H., Bassok, M., Lewis, R., Reimann, P., \& Glaser, R. (1989). Self explanations: How students study and use examples in learning to solve problems. Cognitive Science, 13, 145-182.
CoHen, J. (1992). A power primer. Psychological Bulletin, 112, 155159.

Gentner, D., Rattermann, M. J., \& Forbus, K. D. (1993). The roles of similarity in transfer: Separating retrievability from inferential soundness. Cognitive Psychology, 25, 524-575.

Gick, M. L., \& HOLYOAK, K. J. (1983). Schema induction and analogical transfer. Cognitive Psychology, 15, 1-38.

HOLYOAK, K. J., \& KoH, K. (1987). Surface and structural similarity in analogical transfer. Memory \& Cognition, 15, 332-340.

KAISER, M. K., JoNides, J., \& ALEXANDER, J. (1986). Intuitive reasoning about abstract and familiar physics problems. Memory \& Cognition, 14, 308-312.

LANCASter, J. S., \& Kolodner, J. L. (1988). Problem solving and learning in a natural task domain (Tech. Rep. No. GIT-ICS-89/02). Atlanta: Georgia Institute of Technology.

LeFevre, J., \& Dixon, P. (1986). Do written instructions need examples? Cognition \& Instruction, 3, 1-30.

McCloskey, M., Caramazza, A., \& Green, B. (1980). Curvilinear motion in the absence of external forces: Naive beliefs about the motion of objects. Science, 210, 1139-1141.

MCCloskeY, M., \& KoHL, D. (1983). Naive physics: The curvilinear impetus principle and its role in interactions with moving objects. Journal of Experimental Psychology: Learning, Memory, \& Cognition, 9, 146-156.

McCloskey, M., Washburn, A., \& Felch, L. (1983). Intuitive physics: The straight-down belief and its origin. Journal of Experimental Psychology: Learning, Memory, \& Cognition, 9, 636-649.

Pirolli, P. L., \& ANDERSON, J. R. (1985). The role of learning from examples in the acquisition of recursive programming skill. Canadian Journal of Psychology, 39, 240-272.

Ranney, M., \& ThaGard, P. (1988). Explanatory coherence and belief revision in naive physics. In Proceedings of the 10th Annual Conference of the Cognitive Science Society (pp. 426-432). Hillsdale, NJ: Erlbaum

ReEd, S. K., Ackinclose, C. C., \& Voss, A. A. (1990). Selecting analogous problems: Similarity versus inclusiveness. Memory \& Cognition, 18, 83-98.

Ross, B. (1984). Remindings and their effects in learning a cognitive skill. Cognitive Psychology, 16, 371-416.

Ross, B. (1987). This is like that: The use of earlier problems and the separation of similarity effects. Journal of Experimental Psychology: Learning, Memory, \& Cognition, 13, 629-639.

Ross, B. (1989). Distinguishing types of superficial similarities: Different effects on the access and use of earlier problems. Journal of Experimental Psychology: Learning, Memory, \& Cognition, 15, 456-468.

Thagard, P., Holyoak, K. J., Nelson, G., \& Gochfeld, D. (1990). Analog retrieval by constraint satisfaction. Artificial Intelligence, 46, 259-310.

\section{NOTE}

1. An exception to this is that people aim at a location above the target, knowing that water will fall.

(Manuscript received March 8, 1994; revision accepted for publication May 24, 1994.) 